\title{
MULTIWALENGTH STUDIES OF STAR FORMING REGIONS IN THE MAGELLANIC CLOUDS
}

\author{
MÓNICA RUBIO \\ Departamento de Astronomía, Universidad de Chile \\ ALESSANDRA CONTURSI \\ IPAC, Caltech \\ JAMES LEQUEUX \\ DEMIRM, Observatoire de Paris \\ RODOLFO BARBÁ \\ Observatorio de La Plata
}

RONALD PROBST

$N O A O$

\begin{abstract}
We present a multiwavelength study of several star forming regions in the LMC and SMC. Broad and narrowband IR imaging in conjunction with cold molecular emission of CO lines and mid IR imaging by ISO are providing us the data to define the massive star content and formation processes in low metallicity environments (1/3 to $1 / 10$ solar) for comparison with Galactic star forming regions. Our multiwavelength studies show a clear correlation between the $2.12 \mu \mathrm{m} \mathrm{H}_{2}$, the $6.7 \mu \mathrm{m}$ AIBs, and $230 \mathrm{GHz} \mathrm{CO}(2-1)$ emission as predicted by PDR models towards N66 in the SMC and 30 Doradus in the LMC. We have found IR embedded sources toward the peaks of the $\mathrm{CO}$ emission detected toward both HII regions. We find that the molecular gas that has not yet been photodissociated by the UV radiation field of the $\mathrm{O}$ stars is in hot, dense clumps with very small filling factors. The distribution and morphology of the excited molecular gas in 30 Doradus as seen in the $\mathrm{H}_{2}$ line is clumpy with numerous knots while that of the ionized gas in the $2.16 \mu \mathrm{m} \mathrm{Br} \gamma$ emission shows a filamentary structure. The $6.7 \mu \mathrm{m}$ images show a good correlation to the $\mathrm{Br} \gamma$ emission as expected for warm gas.

Towards N11 and N159 in the LMC we have also found several IR embedded sources associated to the presence of cold molecular gas. These are seen in the interfaces between the molecular material and the HII regions. Thus, either induced or trigered star formation occurs in these interfaces producing a PDR region.
\end{abstract}

Keywords: star formation, IR, Magellanic Clouds

\section{Acknowledgements}

M.R. wishes to acknowledge support from FONDECYT (CHILE) grant 1990881. This work is supported by a Chile-France ECOS grant C97U03. 
